\title{
How to Use the Wisdom of the Crowds to Achieve Innovative Teaching
}

\author{
Xiaoquan Wang \\ School of Business Administration, South China University of Technology, Guangzhou, China \\ Email:201620123814@mail.scut.edu.cn
}

How to cite this paper: Wang, X.Q. (2019) How to Use the Wisdom of the Crowds to Achieve Innovative Teaching. Open Journal of Social Sciences, 7, 346-355. https://doi.org/10.4236/jss.2019.73029

Received: February 27, 2019

Accepted: March 19, 2019

Published: March 22, 2019

Copyright ( 2019 by author(s) and Scientific Research Publishing Inc. This work is licensed under the Creative Commons Attribution International License (CC BY 4.0).

http://creativecommons.org/licenses/by/4.0/

\begin{abstract}
In the postgraduate education stage in China, the curriculum plan and teaching content are only responsible for teachers and experts. There are often deficiencies and professional biases. We propose to adapt the wisdom of the crowds, use social networks as information transmission media to gather insights, opinions and suggestions of the general public diversity to realize innovative teaching. We first expounded the development of the wisdom of the crowds and its connotation, and then proposed a complete process based on the wisdom of the crowds to achieve innovative teaching, and tested the effectiveness and superiority of the process through practice.
\end{abstract}

\section{Keywords}

Wisdom of Crowds, Social Network, Innovative Teaching, Text Mining

\section{Introduction}

China's graduate education industry continues to develop rapidly. Statistics show that from 2013 to 2017, the number of the enrolment of graduate students, the number of postgraduates in university and the number of graduate are increasing year by year. Among them, the number of graduate students enrolled in 2017 is $33.2 \%$ higher than that of 2016 [1]. The continuous expansion of the scale of graduate students, especially in the context of Double First-Class, has put forward higher requirements for the quality of teaching. The traditional indoctrinating teaching mode has been unable to adapt to the development of the times.

Many scholars and experts constantly explore innovative teaching patterns. In terms of teaching methods, Yang Lei and Sun Yanming proposed the on-site teaching in practice base for the master of engineering education [2]. Mo Yongyi assumed that the introduction of teaching methods such as flipping classrooms 
and cooperative learning into English reading can enable students to change from passive recipients of knowledge to active users of knowledge and become the master of the class [3]. In terms of postgraduate training programs, Zhen Liang and Kang Jun proposed to combine the characteristics of different types of graduate students with their training objectives and establish corresponding training systems and assessment criteria, which will help improve the quality of talent training [4]. Wu Ruilin, Wang Jianzhong pointed out the characteristics of graduate teaching based on quality required for innovative graduate students. Simultaneously, they proposed a complete research teaching mode to improve the innovation ability of graduate students [5]. With the development of information technology, micro-curricular courses came into being. Liang Leming and others tried to construct a micro-curriculum model to effectively promote learning based on the comparative analysis of micro-courses at home and abroad [6]. In addition, many colleges and universities continue to develop distinctive teaching methods in order to improve the quality of personnel training. For example, some colleges and universities create a core international curriculum system to improve the level of internationalization, some allow scholars and business experts to jointly design courses to enhance the applicability and practicality of the curriculum and some introduce the curriculum system of foreign excellent universities to perfect the graduate training program.

It is not difficult to find that the theory, methods or practice of these innovative teachings, to a certain extent, enhanced the initiative of students and at the same time improved the classroom experience of students, which has a positive effect on improving the quality of graduate education. However, in the wave of globalization, we must not only pay attention to the teaching experience of students, but also focus on cultivating compound talents with interdisciplinary knowledge background and a cross-disciplinary mindset. On the other hand, social networks such as WeChat and Weibo have penetrated into every aspect of life and become an indispensable tool for the masses to pick up information and handle daily affairs. The huge cardinal number of the social network user and the convenience of operation provide assistance for solving various problems by using the wisdom of crowds. From this point of view, gathering the wisdom of crowds through the social network can integrate the opinions, suggestions and insights of different groups, thus enriching the teaching content, improving the teaching methods, and perfecting the curriculum plan. It has strong operational and positive and far-reaching research significance.

\section{Wisdom of the Crowd (WOC)}

The wisdom of crowds believes that a group has the wisdom to transcend individuals within the group, even if the individuals within the group are not outstanding. Surowiecki's 2005 book, The Wisdom of Crowds, explains the wisdom of the crowds as: In an appropriate environment, groups are intellectually prominent and often smarter than the smartest people in the group. Even if the 
majority of the group is not particularly knowledgeable or rational, they can still make a decision that reflects collective wisdom [7]. This definition is widely accepted. Surowiecki also summed up the four necessary conditions to realize the wisdom of crowds: the diversity of opinions, the independence of decision-making (people's views are not affected by the opinions of the surrounding people), the decentralization of power, and the aggregation mechanism.

The study of the wisdom of crowds has been in existence for a long time. It can be traced back to 1906. In an activity of guessing the weight of an ox, British scientist Francis Galton found that the mean value of the group is almost the same as the real value, and he judged this phenomenon as the wisdom of crowds [8]. Since then, the wisdom of crowds has received more and more attention. From the 1920s to the 1950s, the wisdom of crowds began to be confirmed by a large number of experiments. Hazel Knight, a biologist at Columbia University, conducted a series of studies on the wisdom of crowds, which opened the way for research in this field. For example, she let the students in the class estimate the temperature of the classroom and then calculated the mean of the estimates. The result is only 0.4 degrees from the actual value (the average of the estimates is $72.4^{\circ} \mathrm{F}$ and the actual value is $72^{\circ} \mathrm{F}$ ). Jack Treynor, a finance professor, experimented with a bottle of 850 beans, and the group guessed an average of 871 .

The wisdom of crowds can be widely applied to various fields: politics, economic forecasting, security assessment, public policy assessment, etc. Aspinall $\mathrm{W}$ used the wisdom of the group to predict volcanic eruptions and determine the likelihood and early warning level of volcanic eruptions [9]. Sheng K M Y, Steyvers $\mathrm{M}$ et al. applied the wisdom of crowds for complex combinatorial problems such as Traveling Salesman Problem (TSP), Minimum Spanning Tree Problem (MSTP) [10]. Surprisingly, the application of group wisdom is rarely seen in the field of education [11].

\section{Method}

Using social networks such as WeChat and Weibo to collect insights, opinions, proposals, etc. from different people, that is, group wisdom, can help enrich teaching content, improve teaching methods, and ameliorate curriculum plans, thereby improving the quality of graduate education.

The large number of users of social media can ensure the diversity of opinions and avoid the professional "prejudice" that the teacher group may have due to the similar knowledge background. At the same time, the participants in the social media are separated from each other to ensure the independence of opinions. The diversity of ideas and the independence of decision-making are indispensable conditions for the generation of the wisdom of crowds.

The process of using social networks for innovative teaching based on the wisdom of crowds is shown in Figure 1. First, determine the goal, that is, determine whether the wisdom of crowds is used for teaching content improvement, or for teaching methods, curriculum planning. Then, the project background is 


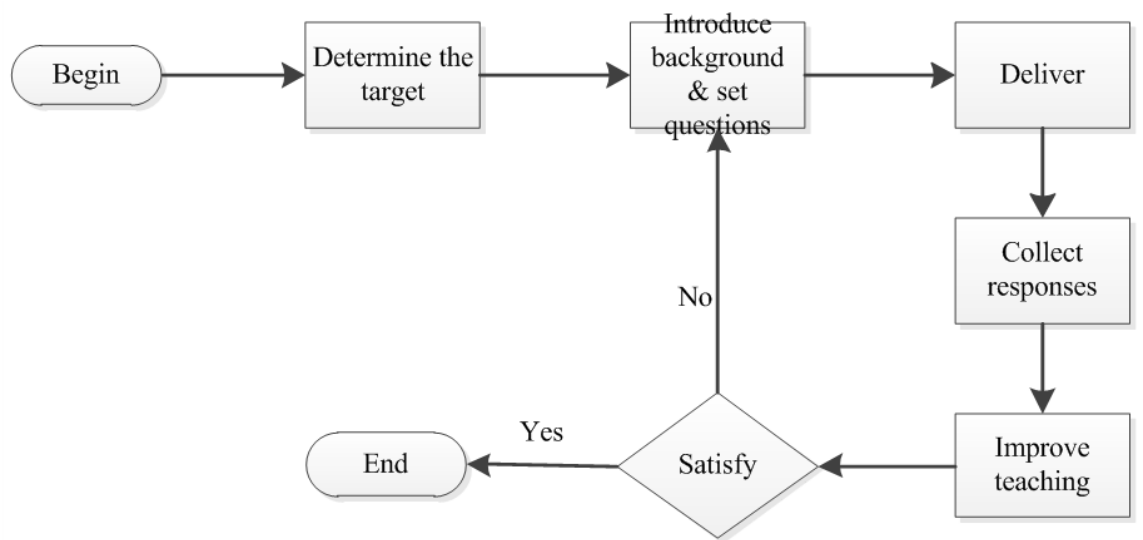

Figure 1. The wisdom of crowds for innovation teaching flow chart.

briefly introduced according to the set goals, so that the participants can enter the situation as soon as possible, and the specific questions are answered by the participants. Next, we will post the questions through social networks such as WeChat, Weibo, e-mail, etc., as much as possible to ensure the diversity of participants (such as participants in different industries, different age distribution and educational background). Finally, the responses received are summarized and analyzed, and the appropriate insights, opinions, and suggestions are used to improve the teaching.

In the stage of data aggregation analysis stage, when the group opinions are small, we can select appropriate suggestions or insights through direct observation. When there are many group opinions, especially the data belongs to unstructured text data, text mining or machine learning and other methods can be used at this time to gain insight into meaningful perspectives. The specific process is shown in Figure 2.

Step 1: Read the text data. Read text data obtained on social networks through Python programming.

Step 2: Word segmentation. Word segmentation is the division of text sequences (sentences, chapters, etc.) into individual words, which is the basis of text mining. The common word segmentation algorithms can be roughly divided into three categories: word segmentation based on string matching, word segmentation based on understanding, and word segmentation based on statistics. Among them, the word segmentation method based on string matching is mechanical segmentation, which matches the text object with a "sufficiently large" dictionary to determine the word segmentation. The statistical segmentation method considers that the probability of the word adjacent to the word can better reflect the credibility of the word. The statistical model is N-gram model and hidden Markov model (HMM). After the end of the word segmentation, judge whether the result of the word segmentation is reasonable. If it is reasonable, proceed to step 4 , otherwise proceed to step 3 .

Step 3: Identify ambiguous words and new words. Ambiguity refers to a variety of segmentation methods for words or sentences, so errors are likely to occur 


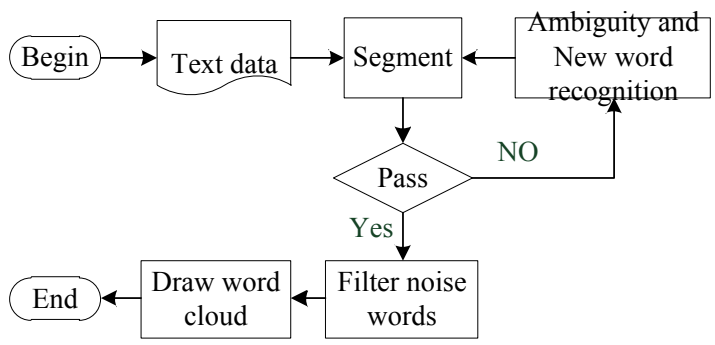

Figure 2. Text mining flow chart.

when segmentation. The new word means that some people's names, place names, professional terms, etc. are not included in the word segmentation dictionary, and it is difficult to identify them when they are cut. These words are also called unregistered words. After ambiguity recognition and new word recognition, proceed to step 2 to perform word segmentation.

Step 4: Filter the noise words. After getting better word segmentation results, we need to filter the noise words, mainly to filter some useless auxiliary words or punctuation.

Step 5: Draw a word cloud. The so-called word cloud is to display words with higher frequency in the text in a more prominent way, so that the viewer can grasp the essence of the text more quickly and grasp the key points of the text.

\section{Empirical Research}

Based on the wisdom of crowds, using social networks to deliver and recycle group insights, and using text mining methods to analyze how the wisdom of crowds can help instructors further enrich teaching content.

\subsection{Data Collection}

The teaching subject is selected as advertising, and the teaching content includes but is not limited to the origin of the advertisement, the concept, the constituent elements, the basic principle, the operation law and so on. The choice of this subject is due to the fact that the subject has professional theories and knowledge in the field. On the other hand, advertising is very common in people's daily lives. Advertising can be seen on media platforms such as TV and movies, as well as on transportation vehicles such as subways, buses. This will not make the participants feel strange or create professional barriers. This will not make the participants feel strange or create professional barriers.

The question is: Advertising is a discipline that tells the origin, composition, basic principles and operation rules of advertising. In order to make the teaching content more interesting and interesting, I would like to introduce some cases into the teaching. What advertisements are you impressed in your daily life? Do you have other opinions or suggestions? In addition to this question, it is necessary to collect some basic information of the participants, such as gender, role (occupation), etc., but does not involve personal privacy information. 
Using WeChat, Weibo, email, etc. as a medium, through the spread of social friends, etc., the problem will be distributed to students, parents, scholars, staff of all walks of life, etc., and the collected responses will be summarized, sorted. Constructive ideas, proposals, etc., will be selected to improve teaching content (Table 1).

\subsection{Data Sorting}

The experiment received a total of 41 responses. Table 2 shows some interesting answers and the gender, role, etc. of the respondents. As you can see from the answers received, the respondents recommended advertisements for different products, such as Coca-Cola and Li Shi Delin. etc., expressed his or her interests such as the history of advertising development, and mentioned new forms of advertising such as small theater advertisements. Through these responses, teachers can integrate the opinions of the group or screen new insights to supplement the content of the teaching. Figure 3 shows the gender ratio of the participants and the distribution of their roles. As can be seen from the figure, there are 17 women and 24 men, and the roles (professionals) of the participants are diverse. The diversity of group members contributes to the generation of group wisdom and the introduction of new insights.

Figure 3 shows the gender ratio of the participants and the distribution of their roles. As can be seen from the figure, there are 17 women and 24 men, and the roles (professionals) of the participants are diverse. The diversity of group members contributes to the generation of group wisdom and the introduction of new insights.

Table 1. Wisdom of crowds enriches teaching content experimental information table.

\begin{tabular}{|c|c|}
\hline Item & Detail \\
\hline Basic Information & Gender, role (occupation) \\
\hline Background & $\begin{array}{l}\text { Advertising is a discipline that tells about the origin, composition, } \\
\text { basic principles and operational rules of advertising. }\end{array}$ \\
\hline & There are many cases in the teaching to enrich the teaching content. \\
\hline Questions & $\begin{array}{l}\text { What are the advertisements in your daily life that impress you? } \\
\text { Do you have other opinions or suggestions? }\end{array}$ \\
\hline Way for spreading & Social platforms or networks such as WeChat, Weibo, and email \\
\hline
\end{tabular}

Table 2. Experimental data collation (partial).

\begin{tabular}{cccc}
\hline Number & Gender & Role & Response \\
\hline 1 & Female & Master & Learn about Thai advertising \\
2 & Female & Teacher & Coca-Cola and Pepsi's advertising campaign \\
3 & Male & Programmer & Theatre advertising. Members can't escape \\
4 & Female & Media worker & Bohemian Rhapsody of the British department store. \\
5 & Male & Bank manager & Li Shi Delin mouthwash, passing a concept
\end{tabular}




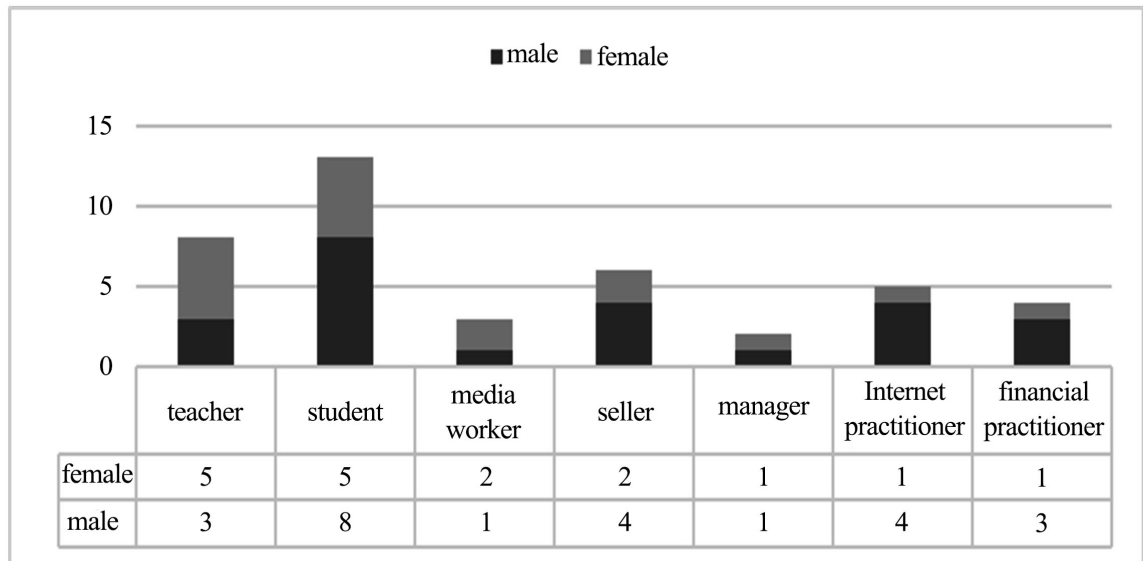

Figure 3. Respondent basic information chart.

\subsection{Data Processing}

1) Data reading: Organize the collected responses into a text file, and use the Python language to program the data in the file. Since the data is in Chinese format, attention should be paid to the encoding conversion during programming.

2) Word segmentation: Since the Python standard library does not contain a word segmentation library, it is necessary to install and import the Jieba library to programmatically implement word segmentation.

3) Ambiguity and new word recognition: In this experiment, some respondents mentioned "Taobao Double Twelve", and after the word segmentation, they were divided into "Taobao/Double/Twelve", and some respondents mentioned "Li Shi Delin mouthwash", the word segmentation algorithm divided "Li Shi Delin" into "Li Shi/Delin", obviously wrong, for this situation, we need to create a user-defined dictionary by programming, including this type of words into the thesaurus. Improve the rationality and correctness of the word segmentation.

4) Noise word filtering: After obtaining good word segmentation results through ambiguity and new word recognition, etc., it is necessary to filter the noise words. For example, after the segmentation, some special punctuation marks will be singular words, and there will be some words that are not helpful for text analysis such as "I", "Yes", "The". For punctuation, it is culled by importing a punctuation library, and for words like "I", "Yes", the word processing method is to exclude words of length 1 .

5) Drawing a word cloud: After doing the word segmentation, draw the word cloud. When programming, you need to download and import the word cloud library. Since the library does not support Chinese, we need to specify in the code that the referenced font is the Chinese font in the computer system font library.

\subsection{Analysis}

Through text mining, the word cloud is shown in Figure 4. It can be seen that 


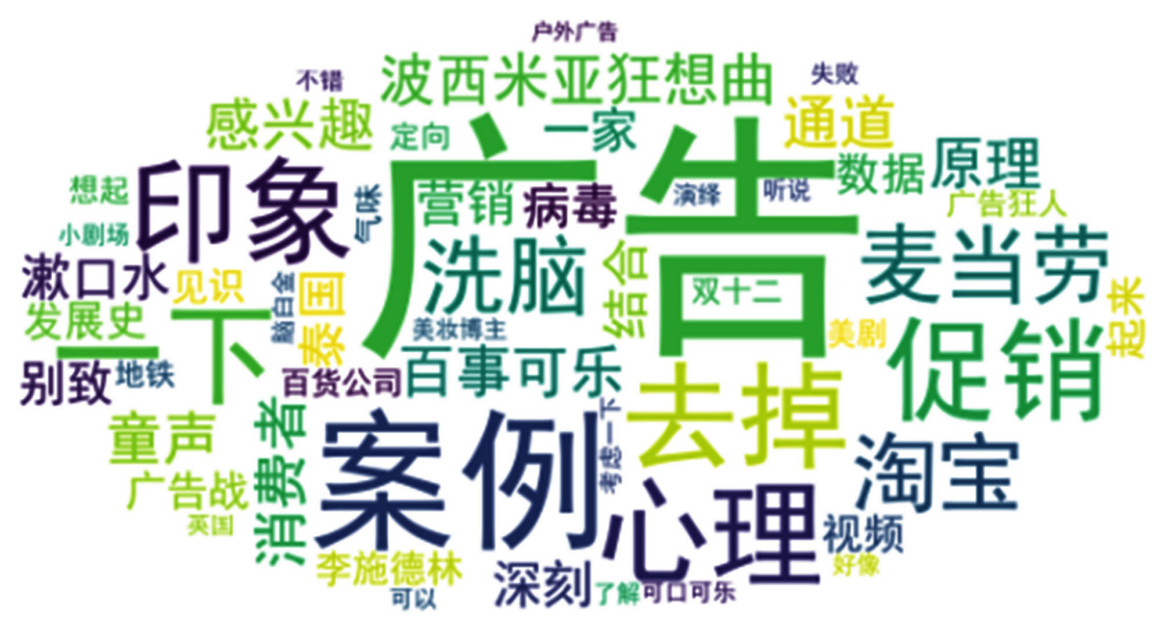

Figure 4. The wisdom of crowds to improve teaching content word cloud map.

the words "advertising" repeatedly mentioned by the respondents are displayed in a very conspicuous manner. Brands such as "McDonald's" and "Li Shi Delin" are also Distinguish by color, size, etc. Moore's Law in the IT field reveals the speed of advances in information technology. Similarly, knowledge is generated and updated all the time, and old knowledge is constantly being eliminated. Even if the latest teaching materials are used and combined with the knowledge and information that the teachers have mastered, the development of teaching content is "backward" and insufficient. This kind of lag and insufficiency is simply irreparable by the individual ability and efforts of the teachers. Through the social network to gather the wisdom of the public, and through the text mining and other means to analyze the formation of sporadic words, insights, often can cover more up-to-date information or insights that teachers forget, neglect, will help the instructor enriches the teaching content and improves the quality of teaching.

The use of the wisdom of crowds not only enriches the content of the lesson, but also discovers interesting, surprising and impressive teaching methods that can be applied to the classroom to enhance the classroom experience of the students. Student experience is a potential component of the quality assurance system for higher education. If the teaching content is regarded as a clear spring, the teaching method is a diversion channel. Only when appropriate guidance can the clear spring of knowledge be delivered to the students' hearts. "Learning skills have their own expertise", and the knowledge reserve of teachers in the professional field is beyond doubt, but it is precisely the accumulation of knowledge in the field of teachers that often forms professional thinking. The way of looking at the problem is inevitably subject to the influence of professional thinking and there is "prejudice", and it is inevitable to be affected in the teaching method. With the wisdom of the group, the teacher will no longer fight alone.

In addition, in the formulation of graduate training programs, the wisdom of crowds can also be used to improve. If the teaching content is regarded as a clear 
spring, the teaching method is a ditches for drainage, and the curriculum plan is the source of water. If the amount of water is too large, it will become a disaster. If it is insufficient, the heart will be exhausted. Therefore, the rationality of the curriculum plan will directly affect the success or failure of teaching. On the other hand, although some scholars have repeatedly called for higher education to be short-sighted and employment-oriented, it is undeniable that teaching still needs to have certain practicability. It cannot be left to be independent, self-respecting, and dissociated from society and contradicts the laws of economic development. From this point of view, improving the practicality of teaching is not necessarily to promote employment, but to cultivate high-quality talents that meet the requirements of the times. It is especially important to focus on the wisdom of the public and listen to the voices of people from all walks of life when developing graduate training programs. These people may be business people who understand what kind of talents the society needs, and may be senior experts who know where the field is headed, and may be the parents of students who have their own expectations for their children. They may be students who have their own plans for the future. If the curriculum plan is developed only by the teaching and research team and experts of the university, it is easy to ignore these factors, and these factors are so important.

\section{Discussion}

As a theory and method of assembling the wisdom of ordinary people, the wisdom of crowds is questioned despite the fact that it is contrary to the theory of a few elites. However, a large number of facts have proved that under certain conditions (guarantee group diversity, independence, etc.), the crowd may produce wisdom that transcends individuals within the group, and this wisdom is not even lost to experts, although individuals within the group are not outstanding. The wisdom of crowds can be applied to various fields such as politics and economic forecasting. In the field of education, the advantages of the wisdom of crowds can also be used to innovate teaching. At the same time, along with the popularity of network and communication equipment, various social media such as WeChat and Weibo have quickly occupied the market with their rich and powerful functions and convenient operations, becoming a national app. The widespread use of these social platforms provides the possibility and convenience of using the wisdom of crowds to innovate teaching.

In today's era of rapid change in knowledge, education and teaching must keep up with the pulse of the times in order not to be eliminated by the times. Experts and teachers are prone to professional thinking "prejudice" because of their similar background knowledge. At the same time, the gap between the age of teachers and students is also very likely to create a "gap" in communication. With social platforms, using the wisdom of crowds, on the one hand, it can produce more diverse insights, opinions or suggestions that are different from domain experts. On the other hand, it can also make up for the teaching of 
teachers, and can incorporate the latest research findings and industry information, which plays an unexpected and vital role in attracting students' interest, improving teaching and improving the quality of postgraduate education.

\section{Conflicts of Interest}

The author declares no conflicts of interest regarding the publication of this paper.

\section{References}

[1] Aspinall, W. (2010) A Route to More Tractable Expert Advice. Nature, 463, 294-295. https://doi.org/10.1038/463294a

[2] Galton, F. (1907) Vox Populi. Nature, 75, 450-451. https://doi.org/10.1038/075450a0

[3] Klieger, A. (2016) The Use of Social Networks to Employ the Wisdom of Crowds for Teaching. Techtrends, 60, 124-128. https://doi.org/10.1007/s11528-016-0020-0

[4] Liang, L.M., Cao, Q.Q. and Zhang, B.H. (2013) Research on Micro-Course Design Pattern-Based on Comparative Analysis of Micro-Courses at Home and Abroad. Open Education Research, No. 2, 65-73.

[5] Mo, Y.Y. (2016) Research on Cooperative Learning Mode under the Concept of Flipping Classroom Teaching. Degree and Postgraduate Education, No. 4.

[6] National Bureau of Statistics (2017) National Annual Statistical Bulletin. http://www.stats.gov.cn/tjsj/tjgb/ndtjgb/

[7] Sheng, K.M.Y., Steyvers, M., Lee, M.D., et al. (2012) The Wisdom of the Crowd in Combinatorial Problems. Cognitive Science, 36, 452-470. https://doi.org/10.1111/j.1551-6709.2011.01223.x

[8] Surowiecki, J. (2004) The Wisdom of Crowds. W. W. Norton \& Company, Inc., New York.

[9] Wu, R.L. and Wang. J.Z. (2013) Research Teaching and Postgraduate Innovation Ability Training. Degree and Postgraduate Education, No. 3, 10-15.

[10] Yan, L., Kang, J. and Ying, S. (2013) Constructing Reform Ideas for Postgraduate Training Model in China. Degree and Postgraduate Education, No. 1, 14-18.

[11] Yang, L., Sun, Y.M. and Chen, X.P. (2014) Innovative Exploration of the On-Site Teaching Course of the Full-Time Engineering Master's Enterprise Practice Base. Degree and Postgraduate Education, No. 2. 